DOI 10.37882/2223-2966.2020.10.25

\title{
ОЦЕНКА ВИДОВОГО СОСТАВА ГРИБНОЙ БИОТЫ НЕКОТОРЫХ ЦЕНОЗОВ, ПОДВЕРЖЕННЫХ АНТРОПОГЕННОМУ В0ЗДЕЙСТВИю
}

\section{EVALUATION OF SOME ANTHROPOGENICALLY AFFECTED CENOSES BY THE SPECIES COMPOSITION OF FUNGI BIOTA}

\section{E. Safaraliyeva A. Safarova K. Bakshaliyeva F. Bayramova G. Balakhanova}

Summary: Were determined that in the formation of mycobiota of various anthropogenically affected areas of Azerbaijan, and licorice and Alhagi maurorum plants growing there participates 102 species of true fungi(Mycota). Became clear that depending on the nature of the anthropogenic impact, the species composition of the mycobiota of the studied areas changes both quantitatively and qualitatively. As a result, regardless from the nature of the anthropogenic impact, occurs decrease in the number of species, and the presence the number of specific species in the structure of the mycobiota varies between 2-7.

Keywords: anthropogenic impact, soil, plant, mycobiota, specific and universal species.

\author{
Сафаралиева Эмиля Мизандар кызы \\ Институт микробиологии НАНА \\ emilya-safara@mail.ru \\ Сафарова Айтен Шамиль кызы \\ Н.с., Институт микробиологии НАНА \\ shahbazova.ayten@mail.ru \\ Бахшалиева Конул Фаррух гызы \\ ә.б.н., Институт микробиологии НАНА \\ konul.baxsh@)mail.ru \\ Байрамова Фериба Васиф гызы \\ Бакинский Государственный Университет \\ feriba.bayramova1990@mail.ru \\ Балаханова Гумру Васиф гызы \\ Азербайджанский Аграрный Университет \\ nesrin.1991@mail.ru
}

Аннотация: Проведенные исследования позволили выявить, что в формировании микобиоты солодки и верблюжьей колючки, произрастающих на различных антропогенно загрязненных территориях Азербайджана, принимают участие 102 вида настоящих грибов (Мусота). Установлено, что в зависимости от характера антропогенного воздействия видовой состав микобиоты исследуемых территорий испытывает как количественные, так и качественные изменения. В результате, независимо от характера антропогенного воздействия, наблюдается уменьшение количества видов, а также наличие в структуре микобиоты специфических видов, количество которых колеблется от 2 до 7.

Ключевые слова: антропогенное воздействие, почва, растения, микобиота, специфические и универсальные виды.

в первую очередь бактерии и грибы, считаются живыми существами, играющими важную роль в процессах деградации растительных остатков, синтезе и деградации гумуса, формировании фитосанитарных условий почвы, накоплении в почве биологически активных веществ, фиксации атмосферного азота и т. д. [9, 14].

Как известно, грибы, являющиеся одним из постоянных компонентов любого ценоза, содержащего органическое вещество, объединяющие в своих рядах как микро-, так и макромицеты, представляют собой организмы, способные распространяться на обширных и разнообразных территориях [8]. Ввиду важности функций, которые они выполняют в местах своего распространения, их изучение является одним из исследовательских направлений, которое остается актуальным на сегодняшний день.

Интенсивное использование для различных целей 
почвенных, водных и лесных экосистем, сформированных на территории Азербайджанской Республики, особенно на Абшеронском полуострове, как небольшой части мира, неизбежно приводит к антропогенному воздействию на эти экосистемы [1]. Несмотря на изучение некоторых из этих экосистем [2], большинство из них требуют сегодня микологической оценки. Поэтому целью данной работы является оценка видового состава грибной биоты серо-бурых почв Азербайджана, подверженных различным антропогенным воздействиям.

\section{Материалы и методы}

Исследования проводились в 2015-2020 гг. на Апшеронском полуострове, а точнее на территории Абшеронского экономического района. Для взятия образцов были выбраны следующие участки (площади 0,75-1,0 га), подверженные различным воздействиям.

1. Территории, загрязненные нефтью и нефтепродуктами;

2. Орошаемые территории

3. Участки, загрязненные автотранспортом, точнее придорожные участки, предназначенные для автотранспорта

4. Территории городского хозяйства, т.е. городские почвы

5. Территории, используемые под свалки

6. Территории, считающиеся относительно чистыми

Образцы были взяты из почв и некоторых растений выбранных территорий (Alhagi maurorum Medik $u$ Glycyrrhiza glabra L.). Выбор этих растений обусловлен тем, что они широко распространены во всех районах Апшеронского полуострова [7] и имеют лечебное значение [11].

С каждого участка было взято по меньшей мере 20 образцов, которые анализировались в соответствии с целью исследования. Отбор пробы, их лабораторный анализ в соответствии с целью исследования, выделение в чистую культуру проводилось в соответствии с методами и подходами, принятыми в микологии $[6,8]$. В качестве питательной среды использовались сусло агар (СА), агар Сабуро, агаризованный Чапек и др. среды. В течение всего процесса отмечаются изменения, связанные с датой образования колонии, формой, цветом, цветом обратной стороны колонии (реверзум), запахом, формой мицелия, образованием конидий и других производных и их формой, размером и т.д. Идентификацию грибов, выделенных в лаборатории, проводили с использованием соответствующих определителей $[10,15]$. Название грибов основано на принципах и подходах международной номенклатуры.

\section{Результаты и их обсужАения}

Сама биосфера, как глобальная экосистема, является одной из сред обитания и питания различных организмов. Изучение грибной биоты как важной составляющей биосферы важно для решения теоретических и практических задач, связанных с биологией в целом, а также медициной, биотехнологией и сельским хозяйством. С этой целью был проведен анализ видового состава микобиоты почв Апшеронского полуострова, подверженных различным антропогенным воздействиям, а также некоторых растений, произрастающих на этих территориях. В результате анализа около 700 образцов (500 из которых почвенные и около 200 - растительные), отобранных в ходе исследований, было идентифицировано 106 видов грибов. Количественные данные, характеризующие их таксономическую принадлежность, приведены в таблице 1.

Таблица 1.

Количественная характеристика таксономической структуры зарегистрированных в ходе исследования грибов

\begin{tabular}{|c|c|c|c|c|c|}
\hline Отдел & Класс & Порядок & Семейство & Род & Вид \\
\hline 3 & 10 & 17 & 28 & 50 & 102 \\
\hline
\end{tabular}

Как видно, хотя все зарегистрированные грибы принадлежат к отделам Zygomycota, Ascomycota и Bazidiomycota царства настоящих грибов (Mycota), их распределение по отделам различается. Так, 11,8\% от общего числа зарегистрированных грибов принадлежат к Zygomycota, 83,3\% - Ascomycota и 4,9\% - Bazidiomycota. Хотя среди выделенных грибов удельный вес сумчатых грибов значительно выше, установлено, что большинство из них относятся к анаморфам.

Следует отметить, что большинство грибов, зарегистрированных в исследованиях, являются известными видами микобиоты, характерными для природы Азербайджана, т.е. идентифицированы в других исследованиях. Однако среди зарегистрированных грибов были обнаружены и виды, впервые зарегистрированные для природы Азербайджана. К этим грибам относятся следующие виды:

1. Ascochyta glycyrrhizae Vasyag

2. Aspergillus ornatus Raper

3. Botrytis longibrachiata Oudem

4. Epicoccum nigrum Link

5. Gliomastix murorum (Corda) S. Hughes

6. Nigrospora sphaerica (Sacc.) E.W. Mason

7. Puccinia glycyrrhizae Rabenh

8. Scopulariopsis brevicaulis (Sacc.) Bainier

9. Talaromyces rugulosus (Thom) Samson

10. Stagonosporopsis cucurbitacearum (Fr.) Aveskamp, Gruyter \& Verkley 
Характеристика видового состава грибов, зарегистрированных на отдельных исследуемых территориях

\begin{tabular}{|c|c|c|}
\hline № & Источник загрязнения & Зарегистрированные виды \\
\hline 1 & Орошаемые воды & $\begin{array}{l}\text { Acremonium charticola, Alternaria alternata, Arthrobotrys oligospora, Aspergillus candidus, A. niger, A. ochraceus, } \\
\text { A.restrictus, A. ustus, A.versicolor, Aureobasidium pullulans, Botrytis cinerea, Candida alpicans, Cephalotrichum } \\
\text { nanum, Circinella circinans, Chaetomium globosum, Cladosporium cladosopides, C.herbarum, C.macrocarpum, } \\
\text { Cylindrocarpon magnusianum, Epicoccum nigrum, Fusarium moniliforme, F.oxysporum, F.solani, F.sporotrichioides, } \\
\text { Mucor circinelloides, M.mucedo, M.plumbeus, Paecilomyces variotii, Penicillium brevicompactum, P.chrysogenum, } \\
\text { P.coprophilum, P.expansum, P.glabrum, P.janthinellum, P.lanosum, P.purpurogenum, P.restrictum, P.rubrum, } \\
\text { P.spinulosum, Rhisobus nicricans, Rh.stolonifera, Scopulariopsis candida, Septoria alhagi, Sporothrix alba, } \\
\text { S.fungorum, Stachybotrys chartarum, Trichoderma asperellum, T.atroviride, Trichothecium roseum, Verticillium } \\
\text { lateritium (50 növ) }\end{array}$ \\
\hline 2 & Нефть и нефтепродукты & $\begin{array}{l}\text { Absidia ramosa, Alternaria alternata, Aspergillus flavus, A. niger, A.ochraceus, A.versicolor, Aureobasidium pullulans, } \\
\text { Botrytis cinerea, Candida alpicans, Circinella circinans, Cladosporium herbarum, Fusarium moniliforme, F.oxysporum, } \\
\text { F. solani, Humicola gricea, Mortierella alpine, Mucor circinelloides, M.hiemalis, Paecilomyces variotii, Penicillium } \\
\text { chrysogenum, P.citrinum, P.cyclopium, P.oxalicum, Puccinia glycyrrhizae, Rhisobus stolonifera, Scopulariopsis } \\
\text { brevicaulis, Torula herbarum, Uromyces glycyrrhizae, Verticillium alboatrum(29 növ) }\end{array}$ \\
\hline 3 & Автотранс-порт & $\begin{array}{l}\text { Absidia ramosa, Acremonium charticola, Alternaria alternata, Aspergillus candidus, A. niger, A. ochraceus, A.repens, } \\
\text { A.restrictus, A.versicolor, Arthrobotrys oligospora, Aureobasidium pullulans, Botryotrichum piluliferum, Botrytis } \\
\text { cinerea, Cercospora glycyrrhizae, Chaetomium globosum, Cladosporium herbarum, Erysiphe alhagi, Fusarium } \\
\text { moniliforme, F.oxysporum, F. solani, F.sporotrichioides, Mortierella alpine, Mucor hiemalis, M.musedo, Myrothecium } \\
\text { roridum, Paecilomyces variotii, Penicillium brevicompactum, P. chrysogenum, P.cyclopium, P.decumbens, P. } \\
\text { expansum, P. janthinellum, P.lanosum, P.oxalicum, P.purpurogenum, P.rubrum, Puccinia glycyrrhizae, Scopulariopsis } \\
\text { brevicaulis, Septoria alhagi, Stagonosporopsis cucurbitacearum, Tichoderma atroviride, Thysanophora penicillioides, } \\
\text { Trichothecium roseum, Uromyces alhagi, U.glycyrrhizae(45). }\end{array}$ \\
\hline 4 & Городское хозйство & $\begin{array}{l}\text { Acremonium atrogriseum, A.charticola, Alternaria alternata, Aspergillus candidus, A.niger, A. ochraceus, A. terreus, } \\
\text { A.versicolor, Cephalotrichum nanum, Circinella circinans, Cladosporium cladosopides, C. herbarum, C.macrocarpum, } \\
\text { Fusarium sporotrichioides, Gliomastix murorum, Mucor circinelloides, M.mucedo, M. plumbeus, Paecilomyces variotii, } \\
\text { Penicillium brevicompactum, P. chrysogenum, P.coprophilum, P.decumbens, P.expansum, P. janthinellum, P.lanosum, } \\
\text { P.purpurogenum, P.rubrum, Phoma glomerata, Ph.herbarum, Rhisobus nicricans, Rh.stolnifera, Sporothrix alba, } \\
\text { Trichoderma atroviride, Trichothecium roseum, Talaromyces rugulosus, Trichophyton terrestre (37) }\end{array}$ \\
\hline 5 & Свалки & $\begin{array}{l}\text { Absidia ramosa, Acremonium charticola, Aspergillus candidus, A. niger, A. ochraceus, A.repens, A.restrictus, A.terreus, } \\
\text { A. ustus, A.versicolor, Aureobasidium pullulans, Botrytis longibrachiata, Cercospora glycyrrhizae, Chaetomium } \\
\text { globosum, Chrysosporinum merdanum, Circinella circinans, Cladosporium cladosopides, C.macrocarpum, } \\
\text { Coniothyrium olivaceum, Cylindrocarpon magnusianum, Erysiphe alhagi, Geotrichum candidum, Fusarium } \\
\text { moniliforme, F.oxysporum, Mucor circinelloides, M.hiemalis, M. mucedo, M.plumbeus, Paecilomyces variotii, } \\
\text { Penicillium brevicompactum, P. chrysogenum, P. citrinum, P. coprophilum, P. expansum, P. janthinellum, P.lanosum, } \\
\text { P.purpurogenum, Pencillium restrictum, P.rubrum, P.spinulosum, Phoma glomerata, Ph.herbarum, Ph.spinasiae, } \\
\text { Puccinia glycyrrhizae, Rhisobus nicricans, Scopulariopsis candida, Sporothrix alba, Stachybotrys chartarum, } \\
\text { Stagonosporopsis cucurbitacearum, Tichoderma atroviride, T. viride, Trichothecium roseum, Trichocladium } \\
\text { polysporum, Uromyces glycyrrhizae, Verticillium dahile(56) }\end{array}$ \\
\hline 6 & Относительно чистые почвы & $\begin{array}{l}\text { Absidia ramosa, Acremonium charticola, Actinomucor elegans, Alternaria chlamydospora, Arthrobotrys oligospora, } \\
\text { Ascochyta alhagi, A.cucumerina, Aspergillus awamori, Aspergillus candidus, A.niger, A.ochraceus, A.ornatus, } \\
\text { A.repens, A.restrictus, A.terreus, A.ustus, A.versicolor, Botrytis longibrachiata, Cephalotrichum nanum, Chaetomium } \\
\text { cellulolyticum, Ch.globosum, Circinella circinans, Cladosporium cladosopides, C.herbarum, C.macrocarpum, } \\
\text { Chrysosporinum merdanum, Colletotrichum brassicicola, Cylindrocarpon magnusianum, Erysiphe alhagi, } \\
\text { Gliocladium roseum, Fusarium oxysporum, F.solani, F.sporotrichioides, Mucorcircinelloides, M.mucedo, M. plumbeus, } \\
\text { Myrothecium roridum, Paecilomyces variotii, Penicillium brevicompactum, P. chrysogenum, P.coprophilum, } \\
\text { Pexpansum, P.janthinellum, P.lanosum, P.purpurogenum, P.rubrum, P.spinulosum, Phoma glomerata, Ph.herbarum, } \\
\text { Pencillium restrictum, Puccinia glycyrrhizae, Rhisobus nicricans, Rh.stolonifera, Scopulariopsis candida, Septoria } \\
\text { alhagi, S.glycyrrhizae, Sporothrix alba, Stachybotrys chartarum, Stagonosporopsis cucurbitacearum, Trichoderma } \\
\text { asperellum, T.atroviride, T.hamatum, T.harzianum, T.viride, Thysanophora penicillioides, Trichothecium roseum, } \\
\text { Uromyces alhagi, U.glycyrrhizae, Verticillium dahile, V.lateritium (71) }\end{array}$ \\
\hline
\end{tabular}


Согласно исследованиям, грибы, впервые зарегистрированные в Азербайджане, составляют 9,8\% от общего количества грибов. Этот факт, а также обнаружение новых видов и в результате других проводимых в Азербайджане исследований, позволяют отметить, что изучение видового состава микобиоты, уникального для природы Азербайджана, пока не находится на желаемом уровне.

Распределение известных, а также впервые идентифицированных видов, зарегистрированных в ходе исследования, неравномерно по отдельным участкам отбора проб (как с почвы, так и с растительности), о чем свидетельствуют данные в таблице 2. Так, из числа зарегистрированных видов грибов на орошаемых землях встречаются 50 видов грибов, на загрязненных нефтью и нефтепродуктами территориях - 29 видов, на придорожных участках для автотранспорта - 45 видов, на территориях городского хозяйства - 37 видов, на свалках - 56 видов, на относительно чистых почвах - 71 вид. Хотя в формировании таких различий участвуют разные виды, и антропогенное воздействие в любом случае приводит к уменьшению видового разнообразия, некоторые виды являются специфическими и встречаются только на определенной территории. Например, количество таких грибов на орошаемых почвах составило 2 вида (Epicoccum nigrum и Sporothrix фунгорум), в почвах, загрязненных нефтью и нефтепродуктами - 3 вида (Humicola gricea, Torula herbarum и Verticillium alboatrum), на территориях, загрязненных автомобильным транспортом - 3 вида (Botryotrichum piluliferum, Myrothecium roridum u Scopulariopsis brevicaulis) на территориях городского хозяйства - 4 вида (Acremonium atrogriseum, Gliomastix murorum, Talaromyces rugulosus и Trichophyton terrestre), на свалках - 3 вида (Coniothyrium olivaceum, Geotrichum candidum и Trichocladium polysporum) и на относительно чистых почвах - 7 видов (Actinomucor elegans, Alternaria chlamydospora, Chaetomium cellulolyticum, Chrysosporinum merdanum, Gliocladium roseum, Trichoderma hamatum və T.harzianum). В зависимости от антропогенного влия- ния некоторые виды по своему распространению были охарактеризованы как космополиты (Aspergillus niger, A. ochraceus, A.versicolor, Paecilomyces variotii и Penicillium chrysogenum), поскольку они были обнаружены в образцах всех 6 участков.

Основными причинами различий в количественых и качественных показателях микобиоты разных территорий могут явиться различия физико-химических характеристик почв (рН, содержание гумуса, влажность и т. др.), а также флоры данных территорий. Это подтверждается результатами ряда исследований.

Как уже отмечалось, образцы были взяты также с солодки и верблюжьей колючки, произрастающих на выбранных участках территории Апшерона. Хотя некоторые из грибов, обнаруженных на этих растениях, зарегистрированы и в почве, взятой из этого участка, в исследованиях встречались и виды, которые могли распространяться только на них. Так, 6 из 27 видов грибов, обнаруженныхна верблюжьей колючке (Ascochyta alhagi, Aspergillus ornatus, Botrytis longibrachiata, Erysiphe alhagi, Septoria alhagi, Uromyces alhagi), и 4 из 23 видов, обнаруженных на солодке (Ascochyta glycyrrhizae, Nigrospora sphaerica, Puccinia glycyrrhizae и Stagonosporopsis cucurbitacearum) обладали специфичностью.

Точнее, в исследованиях они встречались только в образцах, взятых с растений. Однако среди грибов, обнаруженных в образцах верблюжьей колючки и солодки, не было обнаружено никаких видов, специфичных в отношении источника загрязнения.

Таким образом, в формировании микобиоты различных антропогенно загрязненных территорий Азербайджана, а также произрастающих там верблюжьей колючки и солодки, участвуют как специфические, так и универсальные виды настоящих грибов и наблюдается снижение разнообразия микобиоты независимо от источника загрязнения.

\section{ЛИТЕРАТУРА}

1. Алекперов А. Б. Апшерон: проблемы гидрогеологии и геоэкологии. Баку, 2000, 484c.

2. Бахшалиева К.Ф. Анализ аннотированного списка токсигенных микромицетов, распространных на различных ценозах Азербайджана.// Международный научный журнал “SciencerRise"(Украина), 2016, v12, № 1, с. 6-10.

3. Гринченко Т.А., Маклюк Е.И., Журавлева И.М. Биохимическая активность и функционально-структурные особенности микробного ценоза чернозема типичного, загрязненного тяжелыми металлами.//Почвоведение и агрохимия, 2013, № 2(51), с. 134-142

4. Дымов А.А., Каверин Д.А., Габов Д.Н. Свойства почв и почвоподобных тел Воркута // Почвоведение, 2013, №2, с. 240-248.

5. Лукина Н.В., Рязанова С.В. Особенности микоризообразования в техногенных экосистемах//Ккосистемы, их оптимизация и охрана. 2012, вып. 7, c. 261-269.

6. Методы экспериментальной микологии/Под. ред. Билай В.И. Киев: Наукова думка, 1982, 500с.

7. Мехтиева Н.П. Биоразнообразие лекарственной флоры Азербайджана. Баку: «Леттерпрес», 2011, 186 с.

8. Нетрусов А.И., Егорова М.А.,З ахарчук Л.М. и др. Практикум по микробиологии. М. : Издательский центр «Академия», 2005, 608с. 
9. Пивкин М.В., Худякова Ю.В., Кузнецова Т.А., Сметанина 0.Ф., Полохин О.В. Грибы аквапочв прибрежных акваторий Японского моря в южной части Приморского края // Микология и фитопатология, 2005, т. 39, N 6, С. 50-61.

10. Саттон Д., Фотергилл А., Риналди М. Определитель патогенных и условно патогенных грибов. - Москва: Мир, - 2001, - 486с.

11. Akbar Sh.Glycyrrhiza glabra L. (Fabaceae/Leguminosae) (Syns.: G. glandulifera Waldst. \& Kit.; G. hirsuta Pall.; G. pallida Boiss. \& Noe; G. violacea Boiss. \& Noe). Handbook of 200 Medicinal Plants. 2020, v.22, p.963-980.

12. Anderson T.-H., Domschc K.H. Soil microbial biomass: The eco-physiological approach // Soil Biology and Biochemistry, 2010, v.42, p.2039-2043.

13. Domsh K.H., Gams W., Andersen T.H. Compendium of soil fungi. London: Acad. Press., 1993, v. 1, 859p.

14. Khatri N., Tyagi S. Influences of natural and anthropogenic factors on surface and groundwater quality in rural and urban areas, Frontiers in Life Science, 2015, 8:1, p.23-39,

15. Kirk P.M., Stalpers J.A. Dictionary of the fungi, 10th edn. CABI publishing / P.M. Kirk, P. F. Cannon, D. W. Minter.- Wallingford(UK), 2008,600 p.

16. McDonald R.I., Marcotullio P.J., Güneralp B. Urbanization and Global Trends in Biodiversity and Ecosystem Services // Urbanization, Biodiversity and Ecosystem Services: Challenges and Opportunities: A Global Assessment. Eds.: T. Elmqvist et al., 2013, p.31-52.

17. Setoa K.C, Güneralpa B., Hutyra L.R. Global forecasts of urban expansion to 2030 and direct impacts on biodiversity and carbon pools // PNAS(USA), 2012, v.109, № 40, p.16083- 16088

18. Wall D.H., Nielsen U.N., Six J. Soil biodiversity and human health.// Nature., 2015, v.528, p.69-76

\begin{abstract}
○ Сафаралиева Эмиля Мизандар кызы (emilya-safara@mail.ru), Сафарова Айтен Шамиль кызы (shahbazova.ayten@mail.ru), Бахшалиева Конул Фаррух гызы (konul.baxsh@)mail.ru), Байрамова Фериба Васиф гызы (feriba.bayramova1990@mail.ru), Балаханова Гумру Васиф гызы (nesrin.1991@mail.ru).
\end{abstract}

Журнал «Современная наука: актуальные проблемы теории и практики»

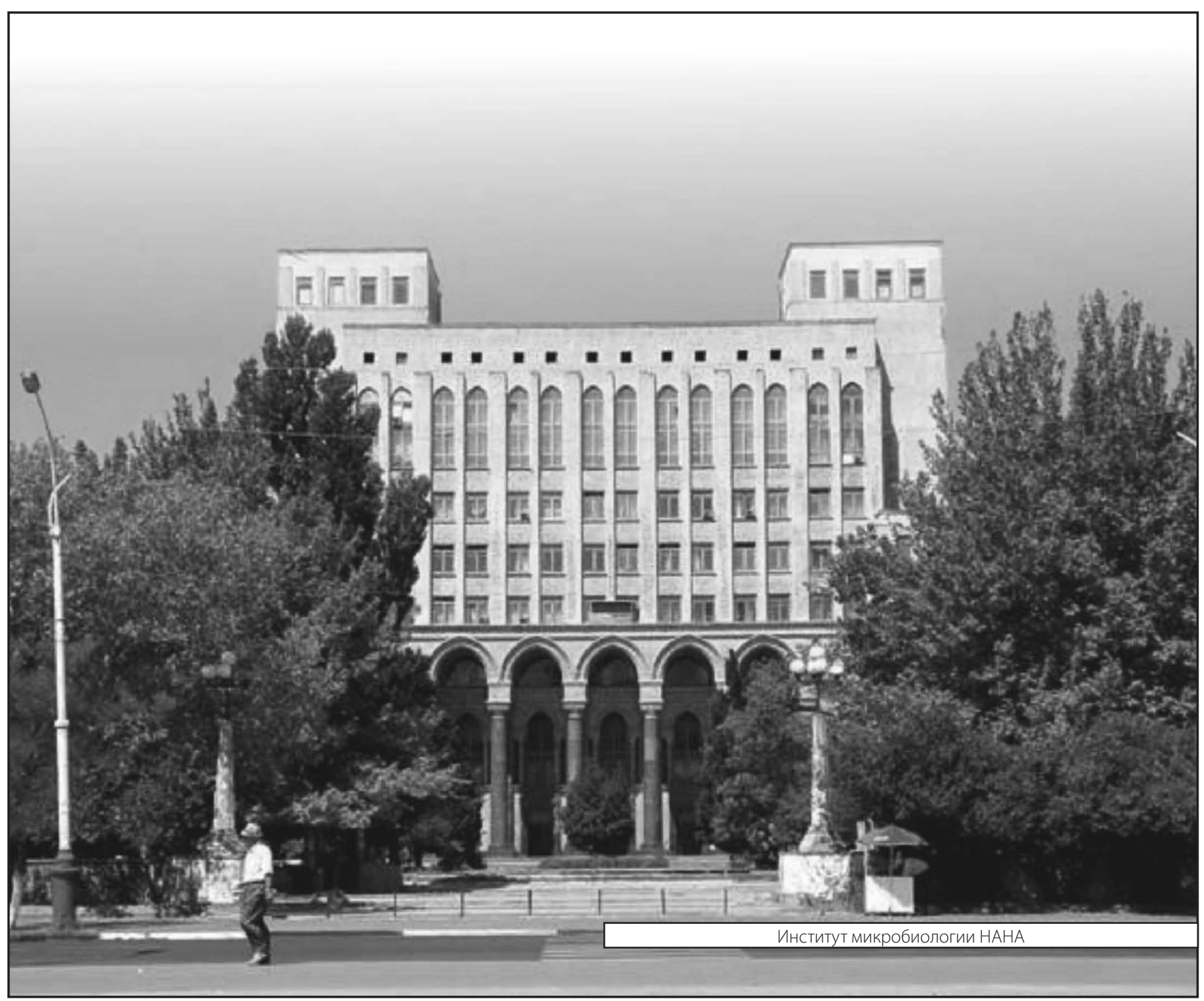

